\title{
Capturing Fluctuations in Pathogen Avoidance: the Situational Pathogen Avoidance Scale
}

\author{
Anastasia Makhanova $^{1}$ (D) E. Ashby Plant $^{2} \cdot$ Jon K. Maner $^{2}$ \\ Received: 4 May 2020 / Revised: 3 August 2020 / Accepted: 5 August 2020 / Published online: 13 August 2020 \\ (C) Springer Nature Switzerland AG 2020
}

\begin{abstract}
Pathogen avoidance is an important motive underlying human behavior and is associated with numerous psychological processes - including biases against social groups heuristically associated with illness. Although there are reliable measurement scales to assess chronic dispositional levels of pathogen avoidance, no measurement scale currently exists to directly assess moment-to-moment fluctuations in pathogen avoidance. This paper presents the Situational Pathogen Avoidance (SPA) scale, which assesses situational variability in pathogen avoidance, especially as it pertains to avoidance of social stimuli. Across six studies, we demonstrate the reliability and validity of the SPA scale, show that the scale is influenced by situational activation of pathogen avoidance motives, and demonstrate that it mediates the association between pathogen avoidance motives (both chronic and situational) and social biases against obese and foreign targets. The SPA scale provides a valuable measurement tool for researchers who study pathogen avoidance and to those who study social biases more generally.
\end{abstract}

Keywords Pathogen threat $\cdot$ Disease avoidance $\cdot$ Behavioral immune system $\cdot$ Social distancing $\cdot$ Scale development

Avoiding pathogens is a key goal for most organisms, including people. Throughout human evolutionary history, coming into contact with pathogens has led to a range of negative consequences, not the least of which is severe illness and death. From avoiding foods that have passed their expiration date to staying away from people with runny noses and coughs, people possess psychological adaptations aimed at helping them steer clear of pathogen threats. The psychology of pathogen avoidance comprises both reactive strategies that regulate behavior when a person perceives an acute pathogen threat and proactive strategies that regulate behavior in the absence of such situational cues (Schaller 2014).

Individual differences in pathogen avoidance are associated with a range of social processes and, as such, have been the focus of significant attention (e.g., Duncan and Schaller 2009;

Electronic supplementary material The online version of this article (https://doi.org/10.1007/s40806-020-00256-8) contains supplementary material, which is available to authorized users.

Anastasia Makhanova

ammakhan@uark.edu

1 Department of Psychological Science, University of Arkansas, 216 Memorial Hall, Fayetteville, AR 72701, USA

2 Florida State University, Tallahassee, USA
Murray and Schaller 2012; Park and Isherwood 2011; van Leeuwen et al. 2017). Indeed, scales intended to measure those individual differences have been developed and used with increasing frequency to test hypotheses pertaining to pathogen avoidance (Duncan et al. 2009; Haidt et al. 1994; Olatunji et al. 2007; Tybur et al. 2009).

Over and above individual differences in pathogen avoidance, the motivation to avoid pathogens can be acutely activated in certain situations. Finding your seat on an airplane only to discover that your neighbor has a nasty cough, for example, is likely to evoke an acute desire to lean away, cover your face, or switch seats. Situationally activated pathogen avoidance motives, like individual differences in pathogen avoidance, can affect a range of social biases (Faulkner et al. 2004; Miller and Maner 2012; Park et al. 2007). Despite the need for a measure that captures situational fluctuations in pathogen avoidance, no such measure currently exists. Individual difference measures of pathogen avoidance typically are not affected by experimental manipulations that increase situational salience of pathogen threat (e.g., Ainsworth and Maner 2014; Makhanova et al. 2015; Makhanova et al. 2019; Mortensen et al. 2010; however, see Sacco et al. 2014). And although researchers can readily measure feelings of disgust, such measures fail to capture the broader experience of pathogen avoidance. 
The current research generates and tests a new scale- the Situational Pathogen Avoidance (SPA) scale - that captures moment-to-moment fluctuations in pathogen avoidance. The SPA scale assesses affective, cognitive, and behavioral responses that comprise pathogen avoidance psychology, especially as it pertains to avoidance of potentially pathogenic social stimuli. Across six studies, we provide evidence for the SPA scale's reliability and construct validity. We show that the SPA scale is responsive to situational cues indicating the presence of pathogen threat. Furthermore, we link the SPA scale to social psychological biases known to reflect activation of pathogen avoidance motives. Our goal is to provide researchers with an essential tool that captures moment-tomoment state-level changes in the psychology of pathogen avoidance.

\section{Pathogen Avoidance Psychology}

Throughout history, pathogens have posed a consistent threat to human health and well-being. Falling ill has many negative consequences, such as experiencing pain, missing out on important opportunities or resources, and the most severedeath. From an evolutionary perspective, those who were better able to avoid illness would have had greater reproductive success compared to those who were less able to do so. Consequently, humans and other species possess powerful psychological mechanisms that mitigate the threat of illness by helping them avoid potential sources of pathogens (Ackerman et al. 2018; Schaller and Park 2011; Tybur et al. 2009).

Early research suggested that disgust plays a key role in helping people avoid illness. Implicated primarily in the realm of ingestive behavior and contamination fears, disgust was shown to promote avoidance of potentially harmful foods and other objects (e.g., Rozin and Fallen 1987). As theories evolved, disgust was also implicated in antipathy toward contact with undesirable people, both those perceived as harboring contagious illness and those perceived as violating moral rules (Haidt et al. 1994). Thus, a long-standing literature suggests that disgust plays a crucial role in self-protection from social threats (for a review, see Tybur et al. 2009). Some theories suggest that acute disgust may be particularly important for in-the-moment, reactive responses to encountering a stimulus perceived as potentially infectious (Schaller 2014). A flurry of recent findings has built on this disgust literature to illuminate the larger psychology of pathogen avoidance.

Pathogen avoidance is a fundamental motive underlying human behavior and promoting a range of important social psychological processes. One of the most well-documented consequences of pathogen avoidance involves social prejudice. For example, people motivated to avoid pathogens tend to display negative biases against immigrants, people with obesity, the elderly, or people who have physical deformities - all groups people tend to heuristically associate with the presence of illness (Ackerman et al. 2009; Faulkner et al. 2004; Miller and Maner 2012; Navarrete and Fessler 2006; Park et al. 2007). Such biases fit with the logic of Error Management Theory (Haselton and Nettle 2006). From this theoretical perspective, people display such biases because the error of assuming something is safe when it is not (a false negative) has more costly consequences than the error of assuming something is dangerous when it is not (a false positive). A fast-growing literature has now linked a range of social biases to pathogen avoidance motives.

The consequences of pathogen avoidance do not end with prejudice. Pathogen avoidance also has implications for morality. People motivated by pathogen avoidance tend to espouse moral world views that help bind the ingroup together to protect the group from internal instability, as well as outgroups who carry novel pathogens to which one's own group has not developed immunity or who may not follow group norms (Murray et al. 2017; Park and Isherwood 2011; van Leeuwen et al. 2017). Motivation to avoid pathogens also seems to affect juror decision-making (Brown et al. 2017). Pathogen avoidance has also been linked with a range of individual differences (e.g., social conservatism, traditionalism, religiosity, conformity) reflecting group-based processes (Murray and Schaller 2012; Terrizzi Jr et al. 2010, 2013; Tybur et al. 2016, c.f. Shook et al. 2015; Tybur et al. 2015a, b) as well as preferences for social interaction partners (Brown and Sacco 2016; Young et al. 2011). Pathogen avoidance has even been tied to basic units of personality such as neuroticism (Tybur et al. 2009; Tybur and de Vries 2013, see also Mortensen et al. 2010).

\section{Individual Differences in Pathogen Avoidance}

A burgeoning literature has linked the aforementioned social phenomena to individual differences in pathogen avoidance. Some people display higher chronic levels of pathogen avoidance than others and, for example, many of the prejudices mentioned earlier appear to be rooted in chronic levels of pathogen avoidance. Individual differences in pathogen avoidance have been implicated in negative biases against people with obesity (e.g., Park et al. 2007), prejudice toward immigrants (e.g., Faulkner et al. 2004), and group-focused moral values (e.g., van Leeuwen et al. 2017).

Individual differences in pathogen avoidance are typically assessed using scales that measure affective, behavioral, and cognitive processes. The field predominantly relies on two such scales: the Perceived Vulnerability to Disease questionnaire (PVD; Duncan et al. 2009) and the Three Domain Disgust scale (TDD; Tybur et al. 2009). The PVD comprises two subscales: germ aversion and perceived infectability. 
Germ aversion focuses on people's tendency to avoid possible sources of pathogens in the environment whereas perceived infectability focuses on chronic cognitions about vulnerability to illness. The TDD examines three social domains (pathogen disgust, sexual disgust, and moral disgust) in which the emotion of disgust tends to be elicited. Pathogen disgust focuses on revulsion in response to stimuli that pose a contamination threat; sexual disgust focuses on revulsion in response to sexual partners and behaviors that deviate from those that would enhance reproductive success; and moral disgust focuses on revulsion in response to others who violate social norms. The PVD and the TDD are widely used and have greatly facilitated the development of the literature on pathogen avoidance. Nevertheless, both scales reflect trait pathogen avoidance and are typically not affected by experimental manipulations of pathogen avoidance motives (e.g., Ainsworth and Maner 2014; Makhanova et al. 2015; Makhanova et al. 2019; Mortensen et al. 2010). Notably, several other scales also tap into trait pathogen avoidance (e.g., Burns et al. 1996; Haidt et al. 1994; Olatunji et al. 2007) but these scales are less frequently used in studies examining the links between pathogen avoidance and social outcomes.

\section{Situational Activation of Pathogen Avoidance}

Although much of the pathogen avoidance research has focused on individual differences, those individual differences are expressed through proximate psychological states and such states are affected by people's current environment. Indeed, independent of their chronic motives, people sometimes find themselves in situations in which there is an immediate threat of pathogens. Such situations can activate acute pathogen avoidance motives. Signs of acute pathogen threat shift people's motivations toward pathogen avoidance in the moment and facilitate psychological processes that mitigate the threat. The acute activation of pathogen avoidance motives is consistent with models of functional flexibility, which suggest that motivational systems come "online" in situations that afford adaptively relevant threats or opportunities (Neuberg et al. 2011). The distinction between chronic and acute pathogen avoidance is also consistent with models of personality that differentiate between dispositional processes and processes that are activated in particular circumstances (e.g., McConnell 2011). Although some people are more chronically avoidant of pathogens than others, acute pathogen avoidance motives should come online in situations that afford an immediate threat of infection.

In fact, situational activation of pathogen avoidance leads to some of the very same psychological processes that have been linked to chronic pathogen avoidance. Situationally activated pathogen avoidance, for example, is associated with social biases against people who display heuristic cues to illness like people with obesity, the elderly, and people of foreign nationality (Faulkner et al. 2004; Miller and Maner 2012). Moreover, situations that increase the salience of pathogen threat promote vigilance to violations of moral values associated with ingroup protection (Murray et al. 2017).

A variety of experimental manipulations have been used to examine psychological processes that reflect situationally activated pathogen avoidance. Some of these manipulations involve participants viewing slideshows of others who are ill (e.g., Lund and Miller 2014; Park et al. 2007), reading articles about the threat of a novel infectious disease (e.g., Miller and Maner 2012; Makhanova et al. 2015), and reading articles that use disease metaphors (e.g., Brown et al. 2019), as well as recalling and verbally describing a time they felt afraid of illness (e.g., Murray et al. 2017). Despite a growing literature examining the effects of such manipulations on social psychological processes, the field lacks a measure that directly captures psychological fluctuations resulting from the situational activation of pathogen avoidance. If research does attempt to quantify situational activations of pathogen threat, this is typically measured by state feelings of disgust. Although feelings of disgust reflect an affective component of pathogen avoidance, simple measures of disgust fail to capture the broader cognitive and motivational aspects of pathogen avoidance. One study attempted to make up for this limitation by altering the beginning of the PVD items to include phrases such as "Currently" and "At this time" (Sacco et al. 2014). In this study, participants who were ostracized, compared to those who were not, reported lower state PVD scores. However, a measure specifically designed to assess situational fluctuations in pathogen avoidance does not exist and is greatly needed.

\section{Current Paper}

The current research develops and validates a scale that captures situational fluctuations in pathogen avoidance: the Situational Pathogen Avoidance (SPA) scale. The SPA scale assesses affective, cognitive, and behavioral responses to hypothetical social situations where pathogen transmission is likely (e.g., "Right now, if someone coughed next to me without covering their mouth, I would move away from them"). To create items, we drew upon the interpersonally focused items from the pathogen disgust subscale of the TDD (e.g., how disgusting is "Shaking hands with a stranger who has sweaty palms") and the germ aversion subscale of the PVD (e.g., "It really bothers me when people sneeze without covering their mouths"). The phrasing of the SPA scale items highlights people's current feelings (rather than typical levels of disgust sensitivity) and behavioral responses to situations where contagion risk is high. Thus, the SPA scale homes in on 
fluctuations in people's pathogen avoidance motives. We did not include items that assess people's general beliefs about their susceptibility to illness. Such items are assessed (at the trait level) with the perceived infectability subscale of the PVD. That subscale assesses cognitions about one's perceived likelihood of becoming ill. Although perceived infectability is a relevant construct in the pathogen avoidance literature, it does not tend to predict social avoidance outcomes as consistently or powerfully as does germ aversion (e.g., Brown et al. 2019; Makhanova et al. 2019; Wang and Ackerman 2019). Moreover, similar to the construct of germ aversion, the focus of the SPA scale is to assess people's affective and behavioral reactions to social situations that are associated with increased contagion risk. Therefore, assessing global perceptions of illness risk is beyond the scope of what the SPA scale is designed to measure.

Study 1 is a preliminary study intended to establish the reliability and factor structure of the scale and examine predictive validity by assessing the association between the SPA scale and aversive reactions toward an obese target (a heuristic cue associated with pathogen avoidance). Next, in 5 studies, we examined whether experimental manipulations of pathogen threat affected scores on the SPA scale. In Study 4, we also provide evidence for convergent and discriminant validity of the SPA scale. Finally, in Studies 5 and 6, we focus on how the SPA scale functions as a mediator between pathogen avoidance (both chronic and situational) and social biases.

\section{Study 1}

Study 1 provides initial evidence for the reliability and the one-factor structure of the SPA scale and examined whether SPA scores were associated with bias against a target linked to heuristic pathogen threat (an obese target). As mentioned previously, past research has demonstrated that pathogen avoidance (both chronic and situationally activated) is associated with prejudice against targets who are obese (Lieberman et al. 2012; Miller and Maner 2012; Park et al. 2007). Evidence for such biases is in line with Error Management Theory (Haselton and Nettle 2006); people display negative biases not just for others who are actually contagious but anyone who possesses cues that heuristically associated with the presence of illness (Schaller and Park 2011). Therefore, examining such biases allowed us to assess the predictive validity of the SPA scale.

\section{Method}

\section{Participants}

Participants $(N=132)$ were students approached by research assistants in common campus locations at a large, public university in the Southeastern US. Research assistants asked participants to answer questions on a (provided) tablet or on their phone, in exchange for candy. One participant was excluded from analyses because they were only 17 years old. The final sample thus included 131 participants $\left(M_{\text {age }}=20.55\right.$, $S D_{\text {age }}=5.41 ; 65 \%$ women; $78 \%$ heterosexual; $63 \%$ White/ Caucasian; 69\% not Hispanic). Participants reported being slightly liberal $(M=6.08, S D=2.19)$ on a 1 (Very Conservative) to 10 (Very Liberal) political orientation scale and moderately religious on average $(M=47.44, \mathrm{SD}=33.48)$ on a 0 (Not religious at all) to 100 (Very religious) scale. Correlations between SPA scores and demographic variables for all studies can be found in the supplemental materials (Table S1). Some demographic variables were included in some studies but not others; we report all that were measured for each study.

\section{Procedures}

Participants were randomly assigned to either complete the SPA first or rate target photographs first (thus, order of presentation was counterbalanced). The SPA scale consists of 10 items $^{1}$ (see Table 1) that focus on affective and behavioral responses to encountering potential pathogens. These items are grounded in past research on the emotion of disgust and draw upon concepts from items in existing scales such as the PVD questionnaire (Duncan et al. 2009) and the TDD scale (Tybur et al. 2009). The SPA scale uniquely highlights responses to encountering a potential social pathogen threat (e.g., "Right now, I would want to wash my hands immediately, if I shook hands with a person with sweaty palms."). This focus on the immediate social context makes the SPA scale valuable for studying social biases and social processes. Furthermore, the focus on pathogen transmission during social interactions is particularly relevant to the study of pathogen avoidance responses because non-zoonotic pathogens (those that can be transmitted human-to-human) may have exerted more pressure throughout human evolutionary history than zoonotic pathogens (those that can infect humans but are not transmitted human-to-human) (see Thornhill et al. 2010). To assess avoidance of zoonotic pathogen sources would have required the addition of a substantial number of additional items, and that was beyond the scope of the current scale. The items in the SPA scale focus on affective (e.g., "Right now, if I was standing next to a person who sneezed I would feel disgusted") and behavioral responses (e.g., "Right now, I would try to sit on the opposite side of the room if I walked into the room where there was a person blowing their nose") and participants are given the following instructions:

\footnotetext{
${ }^{1}$ We started out with 17 items and reduced the number based on fit and itemtotal correlations across all samples. See Supplemental Materials for the 7 excluded items.
} 
Table 1 Situational pathogen avoidance CFA loadings and descriptive statistics

\begin{tabular}{|c|c|c|c|c|}
\hline \multirow[t]{2}{*}{ Item } & \multicolumn{2}{|l|}{ Study 1} & \multicolumn{2}{|c|}{ Combined } \\
\hline & Loading & $M(S D)$ & Loading & $M(S D)$ \\
\hline $\begin{array}{l}\text { 1. Right now, if I was standing next to a person who } \\
\text { sneezed I would feel disgusted. }\end{array}$ & .67 & 3.35 (1.64) & .76 & $3.85(1.75)$ \\
\hline $\begin{array}{l}\text { 2. Right now, I would be grossed out if I shook a stranger's } \\
\text { hand. }\end{array}$ & .55 & $2.03(1.16)$ & .67 & $2.39(1.51)$ \\
\hline $\begin{array}{l}\text { 3. Right now, if someone coughed next to me without } \\
\text { covering their mouth, I would move away from them. }\end{array}$ & .67 & $4.61(1.70)$ & .72 & $4.64(1.74)$ \\
\hline $\begin{array}{l}\text { 4. Right now, it would make me uncomfortable to touch a } \\
\text { door handle in a public restroom. }\end{array}$ & .63 & $3.44(2.09)$ & .66 & $3.52(2.03)$ \\
\hline $\begin{array}{l}\text { 5. Right now, if I heard that a friend had the flu, I would } \\
\text { avoid going to their house or apartment. }\end{array}$ & .41 & $4.23(1.94)$ & .45 & $4.77(1.92)$ \\
\hline $\begin{array}{l}\text { 6. Right now, I would be bothered by sitting in a seat (that } \\
\text { is still warm) that a stranger just got up from. }\end{array}$ & .58 & $3.33(1.76)$ & .52 & $3.24(1.78)$ \\
\hline $\begin{array}{l}\text { 7. Right now, if a person looked like they were sick, I } \\
\text { would be willing to shake their hand. (R) }\end{array}$ & .31 & $4.80(1.63)$ & .45 & $4.98(1.51)$ \\
\hline $\begin{array}{l}\text { 8. Right now, I would be happy to sit close to someone } \\
\text { who just finished an intense workout. (R) }\end{array}$ & .33 & $4.61(1.41)$ & .36 & $4.78(1.50)$ \\
\hline $\begin{array}{l}\text { 9. Right now, I would try to sit on the opposite side of the } \\
\text { room if I walked into the room where there was a person } \\
\text { blowing their nose. }\end{array}$ & .37 & $3.70(1.49)$ & .54 & 3.84 \\
\hline $\begin{array}{l}\text { 10. Right now, I would want to wash my hands } \\
\text { immediately, if I shook hands with a person with sweaty } \\
\text { palms. }\end{array}$ & .64 & $4.14(1.78)$ & .50 & $4.32(1.77)$ \\
\hline
\end{tabular}

The combined column reports data from analyses that combine Study 1 SPA data with those from the control conditions across all studies. These analyses are detailed after Study 6
For this task, you will answer a questionnaire about your attitudes. For the following questions, try to imagine yourself being placed in these situations right now, that is at this moment in time. Please indicate how much you agree or disagree with each of the following statements on a scale of 1 (Strongly Disagree) to 7 (Strongly Agree).

For the target perception task, participants looked at a photograph and imagined what it would be like to interact with the target in the photo-an obese man. ${ }^{2}$ Participants indicated the degree to which they were happy, disturbed, disgusted, creeped out, and would feel uncomfortable if they were in the same room as the target. We averaged the items to create a composite of aversive reactions toward the obese target ( $\alpha=.77$; the happiness item was reversescored).

\section{Results}

The average score on the SPA scale was $3.83(S D=1.03$; range 1.50 to 7 ) and the scale displayed adequate reliability $(\alpha=.79)$. A confirmatory factor analysis assessed the factor structure and model fit (see Table 1 for standardized factor

\footnotetext{
$\overline{2}$ Participants also completed measures to test separate hypotheses that are beyond the scope of the current manuscript.
}

loadings and item-level descriptive statistics). ${ }^{3}$ A one-factor solution resulted in a good-fitting model. The $\chi^{2} / d f$ test, for which values lower than 2 indicate good model fit, resulted in a value of $1.86\left(\chi^{2}=65.04 ; d f=35\right), \mathrm{SRMR}=.064$, $\mathrm{RMSEA}=.084$. Two items had relatively low factor loadings, but these items were reverse-scored (which can reduce reliability but are nevertheless valuable, Barnette 2000; Weems and Onwuegbuzie 2001). Those items displayed higher inter-item correlations in subsequent studies, and we retained them based on theoretical rationale. A two-factor solution (separating affective and behavioral items into different factors) did not result in appreciably better model fit $\left(\chi^{2}=\right.$ 64.96; $d f=34$; SRMR =.064; RMSEA =.086). The chisquare difference test between the two models was not significant, $\chi^{2}(1)=0.08, p=.78$; thus, we retained the one-factor solution. Although the RMSEA indicates only moderate fit (MacCallum et al. 1996), the SRMR is within the recommended .08 cutoff ( $\mathrm{Hu}$ and Bentler 1998). Given recent caution against using fit indices as the only indicators of scale acceptability (e.g., McNeish et al. 2018; Stanley and Edwards 2016), it is important to examine whether the SPA scale functions as intended by responding to experimental manipulation of pathogen threat to judge the acceptability of this scale. We provide those tests in Studies 2 through 6.

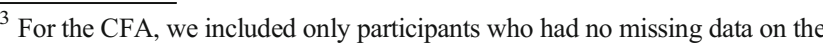
relevant questionnaire items $(n=123)$.
} 
Next, we examined whether the SPA scale had predictive validity by regressing aversive reactions toward the obese target onto SPA scores. SPA scores were significantly associated with aversive reactions toward the obese man, $b=0.22$, $S E=.10, t(128)=2.33, p=.021,95 \%$ CI [0.033, 0.410], semi-partial $r(s r)=.20 .{ }^{4}$ Order of presentation did not affect this association, $b=.05, S E=.19, t(126)=.24, p=.814$.

\section{Discussion}

This study provided preliminary evidence for the reliability of the SPA scale. Additionally, individuals who reported higher (relative to lower) SPA scores also reported more aversive reactions toward an obese target, consistent with prior research linking pathogen avoidance to prejudice against targets heuristically associated with illness.

\section{Study 2}

Our main goal in developing this measure was to create a scale that would be influenced by situational changes in the salience of pathogen threat. In Study 2, we thus conducted an initial test of the hypothesis that SPA scores would be higher when people were exposed to a pathogen threat compared to a nonpathogen threat. Because different experimental manipulations are used in the literature to amplify pathogen threat, it is crucial that any measure of situational pathogen avoidance is generalizable across these manipulations. We thus used two common experimental procedures (an article manipulation and an image manipulation) to heighten pathogen threat. We predicted that regardless of the manipulation used, participants in the pathogen threat condition would score higher on the SPA scale than participants in the matched control condition.

\section{Method}

\section{Participants}

Participants $(N=182)$ were recruited from Amazon's Mechanical Turk and were compensated \$0.25. Based on exclusion criteria established a-priori (see Makhanova et al. 2019), 12 participants were excluded from analyses because they spent fewer than $10 \mathrm{~s}$ viewing the manipulation article. Our final sample included 170 participants $\left(M_{\text {age }}=37.58, S D_{\text {age }}=13.26 ; 60 \%\right.$ women; $76.5 \%$ White $/$ Caucasian). Participants self-reported being fairly liberal $(M=6.14, S D=2.50)$ on a 10 -point Likert scale ( $1=$ "Very Conservative" and $10=$ "Very Liberal") and

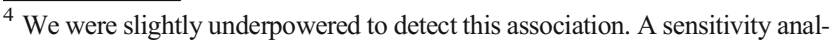
ysis demonstrated that with our sample of 131 participants, we had $80 \%$ power to detect the effect size of $r=.24$.
}

just slightly below the scale midpoint for religiosity $(M=4.35, S D=3.15)$ on a 10 -point Likert scale $(1=$ "Not religious at all" and $10=$ "Very religious").

\section{Procedure}

We used two different procedures to manipulate situational pathogen threat: an article manipulation and an image manipulation. In the article manipulation, participants were randomly assigned to one of two conditions: a pathogen threat article $(n=42)$ or a weather threat article $(n=40)$. Because the study was conducted during the summer, both articles focused on threats that could be heightened during that season (see Makhanova et al. 2019). The pathogen threat article stated that methicillinresistant staphylococcus (MRSA) cases were on the rise and that people should be careful in public areas such as grocery stores, pools, and bars. The control article was about the threat of severe summer weather (e.g., flash floods and fire). In the image manipulation, participants were randomly assigned to view either images of others who were visibly ill $(n=40)$ or of people pointing guns at the camera $(n=48)$. Four photographs for each condition were taken from stimuli used in previous research (see Schaller et al. 2010). Each image appeared on its own page and participants were allowed to click through them at their own pace. We instructed participants to pay attention to the images as they may be asked about them later. In all conditions, immediately after the manipulation, participants completed the SPA scale $(\alpha=.87)$. We conducted a sensitivity analyses using $\mathrm{G}^{*}$ Power for a one-tailed test of the difference between two groups; with our sample size, we would have the power to detect an effect of $d=.38$.

\section{Results}

We examined whether the experimental conditions increased SPA scores relative to the control conditions (see Table 2 for descriptive statistics). We regressed SPA scores onto condition (pathogen threat vs. non-pathogen threat), method of manipulation (articles versus images), and the condition X method of manipulation interaction. Manipulations of pathogen threat significantly increased SPA scores, $b=0.47, S E=$
Table 2 SPA scores in Study 2

\begin{tabular}{lll}
\hline Condition & $M$ & $S D$ \\
\hline Pathogen threat article & 4.45 & 1.30 \\
Weather threat article & 3.97 & 1.28 \\
Pathogen threat images & 4.78 & 1.04 \\
Aggression threat images & 4.32 & 1.13 \\
\hline
\end{tabular}


$0.18, t(166)=2.55, p=.012,95 \%$ CI $[0.11,0.83], s r=.19$, Cohen's $d=.41$. This effect was not moderated by method of manipulation, $b=-0.03, S E=.37, t(166)=-0.08$, $p=.935,95 \%$ CI [-0.757, 0.697], sr $=-.01$.

\section{Discussion}

Findings provide initial evidence that the SPA scale responds to experimental manipulations of pathogen avoidance. This effect emerged across two different manipulations of pathogen threat: one using an article about the threat of an infection and one using photographs of people who were ill. Importantly, the control conditions also represented threats, albeit ones unrelated to pathogens. Thus, SPA scores reflect responses to pathogen threat, specifically, as opposed to physical threats more generally.

\section{Study 3}

In Study 3, we attempted to replicate the results of Study 2 using a slightly different manipulation. We again manipulated pathogen threat with articles, although these articles focused on winter threats (flu and winter weather) to be consistent with the time of year during which the study was conducted.

\section{Method}

\section{Participants}

Participants were undergraduate students $(N=196)$ recruited for an online study through the participant pool of a large, public university in the Southeastern US. We excluded 39 participants based on a-priori criteria. Twenty-two participants spent fewer than $10 \mathrm{~s}$ on the page with the manipulation article (same criterion as in Study 2) and 22 participants answered the catch question embedded in the survey incorrectly ( 5 participants failed both attention checks). Our final sample included 157 participants $\left(M_{\text {age }}=20.01, S D_{\text {age }}=1.40 ; 71 \%\right.$ women; $84.7 \%$ heterosexual; $84.7 \%$ White/Caucasian; $73.9 \%$ not Hispanic). Participants self-reported being fairly moderate $(M=5.53, S D=2.46)$ on 10 -point political orientation scale ( $1=$ "Very Conservative," $10=$ "Very Liberal"). A sensitivity analysis indicated we had $80 \%$ power to detect an effect size as small as $d=.40$.

\section{Procedure}

Participants were randomly assigned to read an article about either the threat of a new avian flu or the threat of severe winter weather (Makhanova et al. 2019). Immediately following the manipulation, participants completed the SPA scale $(\alpha=.84) .^{5}$ Participants also completed several tasks beyond the scope of the current analyses. ${ }^{6}$

\section{Results and Discussion}

An independent samples $t$ test confirmed that participants in the pathogen threat condition scored higher on the SPA scale $(M=4.46, S D=1.00, n=75)$ compared to participants in the severe weather threat condition $(M=3.46, S D=1.00, n=82)$, $t(155)=-6.26, p<.001, d=1.00$. Findings replicated those of Study 2-SPA scores were heightened for participants who read an article about an acute pathogen threat. These results provide further support for the hypothesis that the SPA scale captures fluctuations resulting from the situational activation of pathogen avoidance.

\section{Study 4}

In Study 4, we replicated and extended the findings from Studies 2 and 3 in a laboratory study using a slideshow manipulation of pathogen threat. A third condition was included in which participants washed their hands with a disinfectant hand wipe after encountering a pathogen threat prime. We expected that, although the pathogen prime would increase SPA scores, wiping their hands with the disinfectant wipe would reduce or eliminate that effect (for a similar effect see Huang et al. 2011).

A second goal was to provide evidence for convergent and discriminant validity of the SPA scale. For these purposes, we included a subset of measures used in the development of the PVD questionnaire and the Three Domain Disgust scale (TDD). In the scale development paper for PVD (Duncan et al. 2009), germ aversion was negatively associated with agreeableness, conscientiousness, and positively associated with neuroticism. Germ aversion was unassociated with extraversion, openness to experience, need for cognition, and social desirability. Likewise, in the scale development paper for TDD (Tybur et al. 2009), pathogen disgust was associated with neuroticism, but not the other factors of the Big Five. Thus, in this study, we assessed the associations between SPA scores and the Big Five. We additionally wanted to demonstrate the specificity of the SPA scale for assessing situational fluctuations in pathogen avoidance, so we included state

\footnotetext{
${ }_{5}^{5}$ We also included the Perceived Vulnerability to Disease questionnaire (Duncan et al. 2009). Method and results are reported in the supplemental materials.

${ }^{6}$ Participants read descriptions of two men and responded to questions about how much they would want to engage (or avoid interacting with) each target. These results are reported in the supplemental materials. Participants also completed a measure to test a separate hypothesis that is beyond the scope of the current manuscript.
} 
and trait measures of anger. Finally, because SPA scores should reflect pathogen avoidance specifically, we assessed people's need for cognition and social desirability concerns to demonstrate discriminant validity.

\section{Method}

\section{Participants}

Participants were undergraduate students $(N=170)$ recruited through the participant pool of a large, public university in the Southeastern US. Data from 5 participants were excluded due to issues with following the study protocol (e.g., participant continued to a task before they were instructed to). We excluded 3 additional participants because they incorrectly answered the catch question imbedded in the survey. Our final sample included 162 participants $\left(M_{\text {age }}=18.99, S D_{\text {age }}=1.86\right.$; 85\% women; $91.4 \%$ heterosexual; $74.7 \%$ White/Caucasian; $80.9 \%$ not Hispanic). Participants were fairly moderate in their political orientation $(M=5.53, S D=2.11)$ on a 1 (Very Conservative) to 10 (Very liberal) scale and moderately religious $(M=52.27, \mathrm{SD}=33.63)$ on a 0 (Not religious at all) to 100 (Very religious) scale.

\section{Procedures}

Participants arrived at the lab and were randomly assigned to one of three conditions. In the control condition, participants watched a 5-min slideshow of household objects. In the pathogen threat condition, participants watched a slideshow of others who were visibly ill (Schaller et al. 2010). Both slideshows included ten images shown in random order for $10 \mathrm{~s}$ at a time, with repetition. In the pathogen threat plus wipe condition, participants watched the same pathogen threat slideshow then, as part of an ostensible product rating task, used a disinfectant hand wipe to clean their hands and keyboards (Huang et al. 2011). All participants performed the product rating task in which they rated a pen and a hand wipe on various characteristics. However, only in the pathogen threat plus wipe condition did participants actually use the hand wipe (in the control and pathogen threat conditions the participants only rated the packaging). Participants completed the SPA scale immediately after $(\alpha=.81)$.

Next, participants completed a series of questionnaires including measures of chronic pathogen avoidance. Questionnaires were presented after the manipulation and main dependent measure because we did not want to make pathogens salient for participants in the control condition. Moreover, in studies with similar designs, trait pathogen avoidance measures were not affected by experimental manipulations (e.g., Ainsworth and Maner 2014; Makhanova et al. 2015; Makhanova et al. 2019). The PVD questionnaire includes 15 items that fall into two subscales: Germ Aversion and Perceived Infectability. Germ aversion $(\alpha=.73)$ is related to the desire to avoid potential pathogens in one's environment (e.g., "I don't like to write with a pencil someone else has obviously chewed on") and perceived infectability $(\alpha=.91)$ is related to one's general perception about the susceptibility of one's immune system to disease (e.g., "If an illness is going around, I will get it"). Participants rated their agreement with each statement using a 7-point scale ( 1 = "Strongly Disagree," 7 = "Strongly Agree").

The TDD scale includes 21 items that fall into three subscales: Pathogen Disgust, Sexual Disgust, and Moral Disgust. Pathogen disgust $(\alpha=.77)$ is associated with feelings of disgust elicited by objects in the environment that pose contagion risk (e.g., "Sitting next to someone who has red sores on their arm"). Sexual disgust ( $\alpha=.81)$ is associated with disgust elicited by undesirable sexual encounters (e.g., "Hearing two strangers have sex."). And moral disgust $(\alpha=.89)$ is associated with disgust elicited by violations of social norms (e.g., "Shoplifting a candy bar from a convenience store". Participants rated their agreement with each statement using a 7-point scale (1 = "Strongly Disagree," 7 = "Strongly Agree").

Participants also completed the Ten Item Personality Inventory (TIPI; Gosling et al. 2003), which is a short measure of the BIG-Five personality traits that includes two scale items each for neuroticism, extraversion, openness to experience, conscientiousness, and agreeableness. The need for cognition scale ( $\alpha=.81$; Cacioppo et al. 1984) included 19 items (e.g., "I appreciate opportunities to discover the strengths and weaknesses of my own reasoning") and assessed the trait enjoyment of thinking as an activity. We used the balanced inventory of desirable responding ( $\alpha=.78$; Paulhus 1998) as a measure of social desirability that included 40 items (e.g., "I don't care to know what other people really think of me"). For both of these scales, participants rated their agreement with each item on a 7 -point scale $(1=$ "Strongly Disagree," $7=$ "Strongly Agree").

We adapted state and trait anger questions from existing scales. For the state anger measure, participants answered 8 questions ( $\alpha=.85$; e.g., "I feel angry") using a 7-point scale ( 1 = "Strongly Disagree," 7 = "Strongly Agree"). For the trait anger measure ( $\alpha=.91)$, participants used the same scale to answer 29 questions (e.g., "Once in a while I can't control the urge to strike another person").

Participants also completed two measures of bias against groups heuristically associated with illness. Participants completed the ethnocentrism questionnaire $(\alpha=.81$; the Revised Generalizable Ethnocentrism scale, Neuliep and McCroskey 1997), which included 24 items (e.g., "Most other cultures are backward compared to my culture"). Participants also completed an explicit Anti-Fat measure ( $\alpha=.81$; Crandall 1994), which consisted of 13 items (e.g., "I really don't like fat people much"). Participants rated their agreement with each item 
on a 7-point scale $(1=$ "Strongly Disagree," $7=$ "Strongly Agree"). ${ }^{7}$ At the end of the study participants provided demographic information and were debriefed.

\section{Results}

First, we examined effects of the manipulations on SPA scores. We regressed SPA scores onto two dummy coded condition variables (with the control condition serving as the reference group). The omnibus test confirmed the presence of differences in mean SPA scores across the three conditions, $F(2,159)=3.05, p=.050$. Compared to those in the control condition $(M=3.88 ; S D=0.97)$, participants in the pathogen threat condition reported higher SPA scores $(M=4.32 ; S D=$ $0.95), b=0.44, S E=0.19, t(159)=2.25, p=.026,95 \% \mathrm{CI}$ $[0.05,0.82], s r=.18$. Participants in the pathogen threat plus wipe condition $(M=4.28 ; S D=1.13)$ also had higher SPA scores than the control condition, $b=0.39, S E=0.20$, $t(159)=2.00, p=.047,95 \%$ CI $[0.01,0.78], s r=.16$. Participants in the pathogen threat plus wipe condition, however, did not differ significantly in SPA scores from those in the no wipe pathogen threat condition, $b=0.04, S E=.20$, $t(159)=0.22, p=.827$. Thus, it appears that the experimental manipulation heightened people's situational pathogen avoidance, but cleaning ones' hands with a cleansing wipe did not reduce the effect of the manipulation.

Next, we examined whether SPA scores were correlated with other scales that previously have been shown to be associated with pathogen avoidance. Correlations among all variables are presented in Table 3. SPA scores were positively associated with all three scales of trait pathogen avoidance: germ aversion, perceived infectability, and pathogen disgust $[b=.52, S E=.08, t(157)=6.28, p<.001, s r=.43$, when controlling for moral and sexual disgust]. SPA scores were further marginally positively correlated with neuroticism $(p=.067)$. SPA scores were not associated with other factors of the Big Five. SPA scores were also positively associated with trait, but not state, anger. Moreover, as predicted, SPA scores were not associated with the need for cognition or social desirability scales.

Next, we examined whether SPA scores, as well as measures of trait pathogen avoidance, were associated with ethnocentrism and prejudice against obese people. As predicted, SPA scores were positively associated with ethnocentrism, $r=.16, p=.048$, whereas the associations between ethnocentrism and germ aversion $(r=.11, p=.160)$, perceived infectability $(r=-.04, p=.630)$, and pathogen disgust $(r=.12, p=.136)$ were not significant. However, SPA scores were not associated with prejudice against obese people, $r=.10, p=.189$. Although neither subscale of the PVD was

\footnotetext{
${ }_{7}^{7}$ Participants also completed a measure to test a separate hypothesis that is beyond the scope of the current manuscript.
}

associated with prejudice against obese people (germ aversion: $r=.09, p=.278$; perceived infectability: $r=.12$, $p=.129)$, a significant association emerged with pathogen disgust $(r=.19, p=.017)$. The experimental manipulation of pathogen avoidance did not affect ethnocentrism, $F(2,159)=$ $0.42, p=.660$, or prejudice against obese people, $F(2,159)=$ $0.49, p=.614$.

\section{Discussion}

Study 4 conceptually replicated Studies 2 and 3 and demonstrated that SPA scale scores are increased by an experimental pathogen threat prime. Participants in the pathogen threat conditions reported higher SPA scores than those in the control condition; however, the hand wipe manipulation did not reduce SPA scores. We also observed evidence for convergent and discriminant validity of the SPA scale. That is, the SPA scale was correlated with all three assessments of trait pathogen avoidance (germ aversion, perceived infectability, and pathogen disgust), marginally correlated with neuroticism, and was not correlated with social desirability or need for cognition.

In this study, we also assessed whether SPA scores predicted measures of social bias. Individuals who scored higher on the SPA scale, compared to those who scored lower, reported higher levels of ethnocentrism. However, SPA scores were not associated with an explicit measure of anti-fat prejudice. In Study 1, we examined social bias against an obese target using a measure that focused on aversive reactions toward a target in a photograph. It could be that the SPA scale is more closely associated with affective responses than the more cognitive responses captured by the explicit prejudice scale used in the present study, which assessed perceptions of ability, agency, anticipated social decision-making, and personal worries about own potential weight-gain. Moreover, whereas the SPA scale predicted responses to an image of an individual obese target, it did not predict responses to questions about the obese at the more abstract group level. Thus, situational pathogen avoidance may be more closely tied to reactions to an individual obese target rather than fat people as a group more generally. Consequently, we reverted back to the photographbased measure for the next two studies as we continued to examine the association between the SPA scale and social biases.

\section{Study 5}

Study 5 examined whether the associations between pathogen avoidance - both trait levels of and situational activations of pathogen avoidance - and social biases are statistically mediated by situational pathogen concerns, as measured with the SPA scale. We once again examined aversive reactions 
30

Evolutionary Psychological Science (2021) 7:21-38

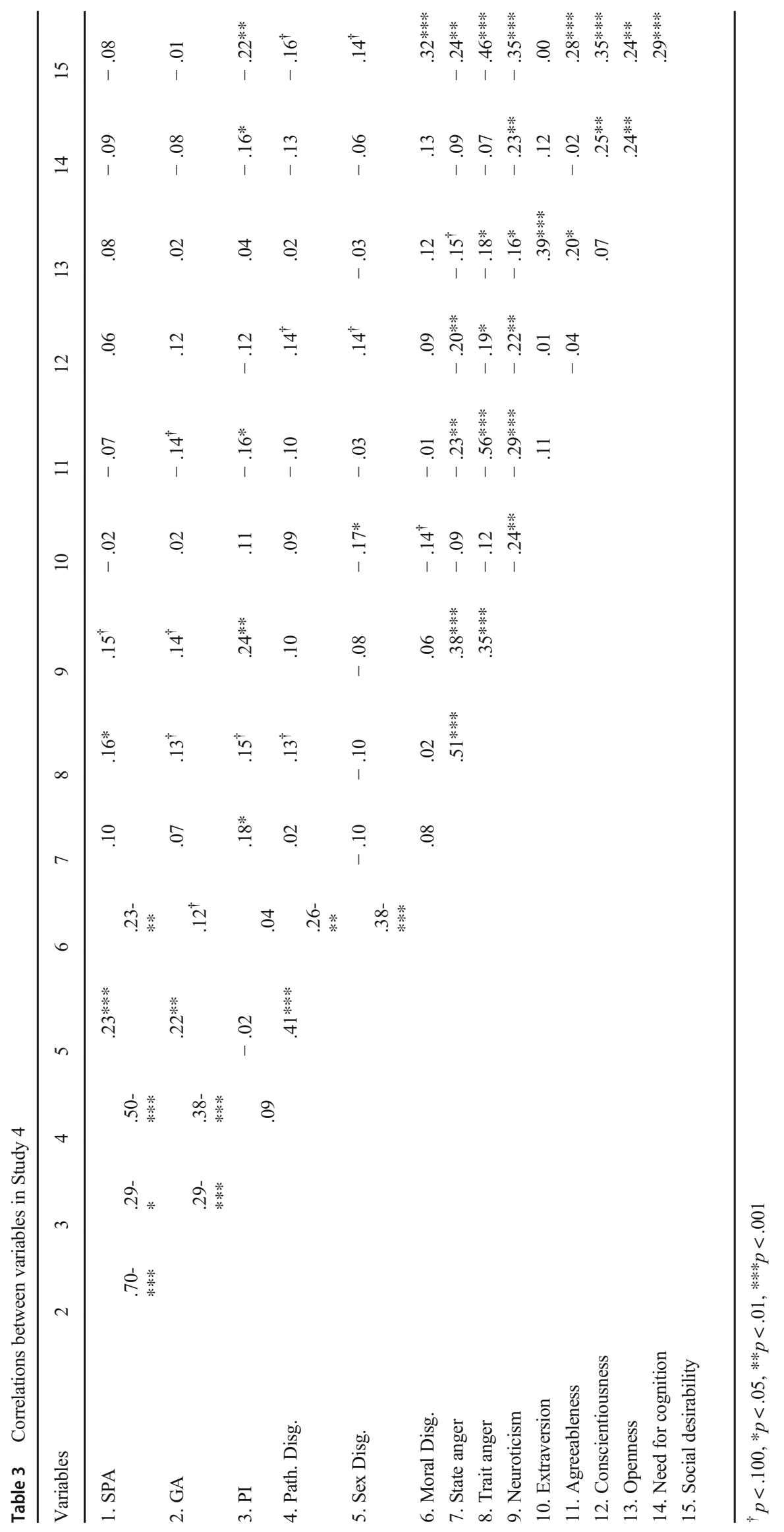

Springer 
toward an obese target (an example of a heuristic cue associated with pathogen avoidance). We also included a non-selfreport measure of ethnocentrism (Oaten et al. 2017) in which participants saw faces of men of different races who held neutral facial expressions and were asked to identify how likely they thought they would be to catch an illness from each target. In previous work, White individuals who had a chronic health condition that decreases immune system function (rheumatoid arthritis), and thus increased susceptibility to pathogens, were more likely to perceive non-White targets to be likely to transmit illness than White targets (Oaten et al. 2017). Theories have suggested that avoidance of groups perceived to be foreign may be adaptive for various reasons such as the threat of novel pathogens (Fincher and Thornhill 2012), wariness of foreign norms (Karinen et al. 2019), lower trust of social partners (Aarøe et al. 2016), and as mentioned previously, heuristic association with disease (Faulkner et al. 2004; van Leeuwen and Petersen 2018). We predicted that SPA scores would statistically mediate any effects of trait pathogen avoidance and experimental manipulation of pathogen threat on these two social biases.

\section{Method}

\section{Participants}

Participants were undergraduate students $(N=370)$ recruited through the participant pool of a large, public university in the Southeastern US. ${ }^{8}$ We excluded 78 participants based on apriori criteria. Fifty-seven participants spent fewer than $10 \mathrm{~s}$ on the page with the manipulation article and 21 additional participants either did not correctly answer the catch question embedded in the survey or did not correctly identify the topic of the article they read. Our final sample included 292 participants $\left(M_{\text {age }}=19.06, S D_{\text {age }}=1.26 ; 74.3 \%\right.$ women; $92.5 \%$ heterosexual; $81.2 \%$ White/Caucasian; $75.0 \%$ not Hispanic). Participants were fairly moderate $(M=5.52, S D=2.25)$ in their political orientation on 1 (Very Conservative) to 10 (Very Liberal) scale.

\section{Procedure}

The study was conducted online. Participants were randomly assigned to read an article about either the threat of a new avian flu or the threat of severe winter weather. This manipulation was identical to that used in Study 3. Following the manipulation, participants completed the SPA scale, PVD questionnaire, and state anger scales. ${ }^{9}$ Because pathogen

\footnotetext{
${ }^{8}$ We preregistered the sample size, exclusion criteria, and study design on the Open Science Framework. Because condition did not predict the SPA scale in this study, we could not conduct some of the analyses we preregistered.

${ }^{9}$ Analyses for state anger are reported in supplemental materials.
}

disgust and germ aversion typically demonstrate similar associations with measures related to prejudice (e.g., Makhanova et al. 2019) and because we switched from the Anti-Fat prejudice scale used in Study 4, we only included the PVD questionnaire in order to limit the length of the online questionnaire.

Participants also performed two photo-rating tasks. First, participants completed the task described in Study 1, in which participants responded with their gut feelings about an obese target. ${ }^{10}$ The second photo-rating task was adapted from Oaten et al. (2017). Participants saw photos of 12 men (3 White, 3 Black, 3 Asian, and 3 Latino) who displayed neutral facial expressions. Faces were selected from the Chicago Face Database and were rated similarly on a variety of attributes. Participants were given the following instructions:

For the next task, you will see a variety of faces. These individuals were instructed to hold a neutral facial expression while being photographed and not show how they might be feeling. Some of these individuals were ill at the time their photo was taken. We are interested if people are able to pick up on any subtle cues even when individuals are holding neutral facial expressions. Please look at each photo and answer the question below.

Then, participants responded to the question "How likely are you to catch a disease from this person?" on a scale of 1 (Not likely at all) to 7 (Very likely). We hypothesized that nonWhite targets would be perceived as more contagious than White targets. The presentation of the state scales (SPA and anger) and the photo-rating tasks was counterbalanced. All participants completed the PVD last. Finally, participants reported demographics.

\section{Results}

First, we examined whether SPA scores were responsive to both chronic pathogen avoidance and situationally activated pathogen avoidance. We regressed SPA scores onto germ aversion and experimental condition. Germ aversion significantly predicted SPA scores, $b=.77, S E=.05, t(289)=16.16$, $p<.001,95 \%$ CI $[0.68,0.86], s r=.69$. However, condition did not predict SPA scores, $b=-0.03, S E=.09, t(289)=-$ $0.30, p=.767,95 \% \mathrm{CI}[-0.21,0.16], s r=-.02$. An independent samples $t$ test confirmed that SPA scores in the pathogen threat condition $(M=4.11, S D=1.06)$ did not differ from those in the control condition $(M=4.01, S D=1.11)$, $t(290)=-0.82, p=.415$, Cohen's $d=.09$.

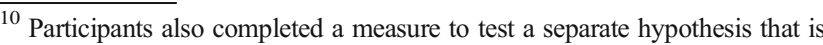
beyond the scope of the current manuscript.
} 
Fig. 1 The models depict mediational analyses from Study 5. Models control for experimental condition and counterbalancing order. The model in panel A uses the whole sample $(n=292)$. The model in panel B uses a subsample of participants who self-reported being White or Black $(n=261)$. This model additionally controlled for contagion risk from non-immigrants (White and Black targets) when estimating effects for contagion risk from immigrants (Asian and Latino). Values are unstandardized coefficients

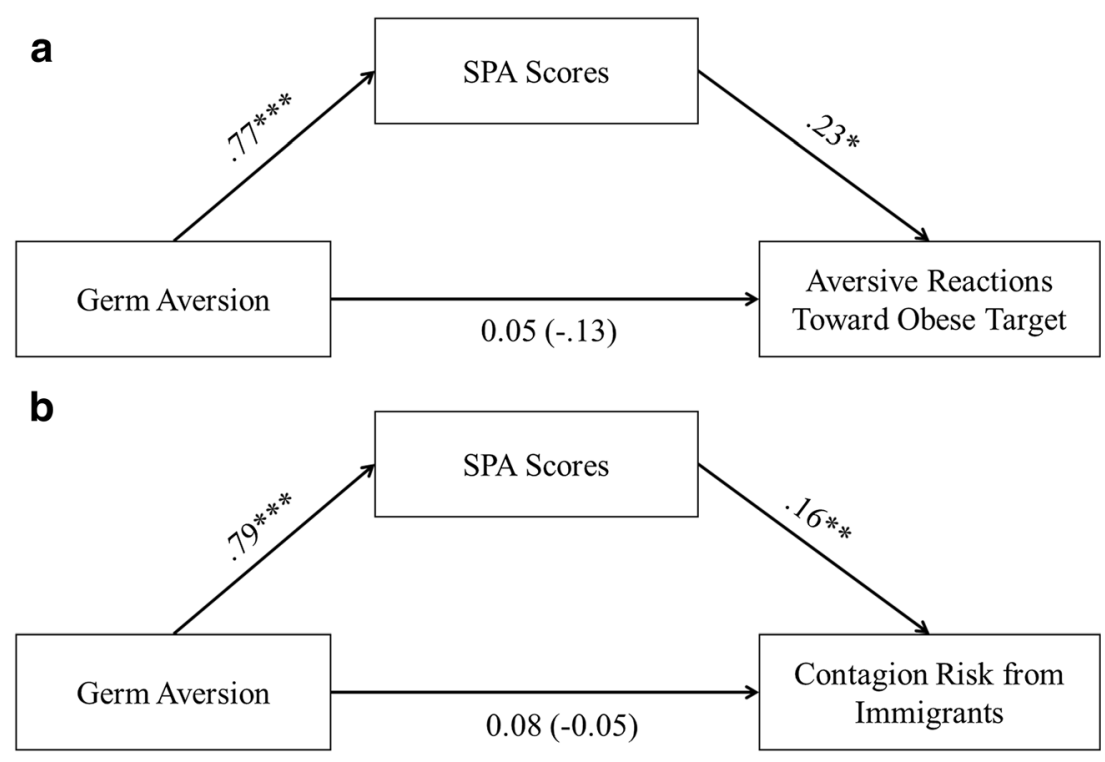

Notably, the photo-rating tasks required all participants to think about catching illnesses, and this could have affected SPA scores for participants who performed that task before completing the SPA. Therefore, we conducted exploratory analyses focused only on participants who completed the SPA immediately after the manipulation. Results indicated that participants in the pathogen threat condition $(M=4.13$, $S D=0.99)$ tended to report higher SPA scores than participants in the control condition $(M=3.90, S D=1.06)$ although this trend was not significant, $t(149)=-1.38, p=.169$, Cohen's $d=.22$.

Because condition did not predict SPA scores, the remainder of our analyses included experimental condition only as a covariate. First, we examined whether the effect found in Study 1 was replicated in this sample by regressing aversive reactions toward the obese target onto SPA scores, controlling for condition and order of presentation. Higher SPA scores were associated with more aversive reactions toward the obese target, $b=0.15, S E=0.07, t(288)=2.26, p=.024,95 \% \mathrm{CI}$ $[0.02,0.28], s r=.13$. In a similar model, GA was not associated with aversive reactions toward the obese target, $b=.05$, $S E=.07, t(288)=0.67, p=.506$. Although there was no direct effect, we examined whether SPA served as a mediator of the indirect effect between GA and aversive reactions toward the obese target. We used PROCESS to explore this association, controlling for condition and counterbalancing order (see Fig. 1a). As presented above, GA scores significantly predicted SPA scores. In addition, SPA scores predicted aversive reactions toward the obese target, $b=0.23, S E=.09$, $t(287)=2.49, p=.013,95 \%$ CI $[0.05,0.41]$, controlling for $\mathrm{GA}$, order of presentation, and condition. The indirect effect of chronic pathogen avoidance through SPA scores was significant, $b=0.18, S E=0.08, p<.05,95 \%$ CI $[0.02,0.32]$. Notably, condition also emerged as a significant predictor of aversive reactions toward an obese target, $b=.38, S E=.14$, $t(287)=2.62, p=.009,95 \%$ CI $[0.09,0.66]$.

Next, we examined whether SPA scores were associated with thinking that neutral targets who were perceived to be foreign harbored a contagious illness. Previous work using this measure found that White targets were rated as less likely to spread contagion than non-White targets but used the analytical strategy that first tested groups for differences within the non-White targets ${ }^{11}$ (Oaten et al. 2017). Thus, following those guidelines, we first examined whether there were any differences in the associations between the SPA scale and the three non-White targets. A repeated measures general linear model indicated that there were significant differences in these associations, $F(2,289)=5.61, p=.004$. The association between SPA scores and perceptions of contagion was significant for both Latino targets $(r=.18, p=.002)$ and Asian targets $(r=.14, p=.020)$ but not Black targets $(r=.03, p=.605)$. Thus, it may be that the cultural context for our American sample is different from that of the Australian sample in the original study. All three non-White targets would likely be viewed as immigrants in Australia. However, in the USA, it is possible that only Asian and Latino targets would be viewed as immigrants whereas Black targets may be perceived as nonimmigrants. Furthermore, this distinction is in line with prior research suggesting that pathogen avoidance biases target groups perceived as relatively more foreign and unfamiliar (Faulkner et al. 2004). Thus, we examined whether SPA scores were associated with perceptions of contagion for immigrant (composite of Asian and Latino) versus nonimmigrant (composite of White and Black) targets. We ran

\footnotetext{
${ }^{11}$ Oaten and colleagues used Black, Southeast Asian, and Chinese Asian as their non-White groups. We substituted Latino targets instead of the Southeast Asian targets.
} 
analyses on a subsample of participants who self-reported being either White or Black $(n=261)$. We regressed perceptions of contagion from immigrants onto SPA scores, controlling for condition, order of presentation, and perceptions of contagion from non-immigrant targets. SPA scores were significantly associated with relative perceived contagion from immigrant targets, $b=.13, S E=.04, t(256)=3.17, p=.002$, $95 \%$ CI $[0.05,0.21], s r=.12$. SPA scores were not associated with perceived contagion from non-immigrant targets, $b=-$ $0.06, S E=.04, t(256)=-1.51, p=.132,95 \%$ CI $[-0.13$, 0.02 ], $s r=-0.06$.

In a similar model that examined associations between GA and perceptions of contagion from immigrant targets, we did not find a direct effect, $b=.08, S E=.05, t(256)=1.64$, $p=.103$. Nonetheless, we examined whether SPA scores served as a mediator of the indirect effect between GA and perceptions of contagion from immigrant targets. We used PROCESS to conduct the analyses of the indirect effect of GA onto perceptions of immigrant targets through SPA scores, controlling for condition, order of presentation, and perceptions of non-immigrant targets (see Fig. 1b). In path a, GA significantly predicted SPA scores, $b=0.79, S E=.05$, $t(256)=15.78, p<.001$. In path $\mathrm{b}$, SPA scores significantly predicted perceptions of contagion in immigrant targets, $b=.16, S E=.06, t(255)=2.82, p=.005$. There was a significant indirect effect through SPA scores, $b=.13, S E=.05$, $p<.05,95 \%$ CI $[0.04,0.22]$.

\section{Discussion}

In this study, we found mixed support for our hypotheses. We replicated the association between chronic pathogen avoidance and SPA scores. Unlike Studies 2-4, however, in Study 5, the experimental manipulation of pathogen avoidance did not affect SPA scores. Notably, the presence of non-significant results is expected in a set of studies (Lakens and Etz 2017). Thus, we reported these results to increase transparency and conducted a within-manuscript meta-analysis of the effects of experimental manipulations on SPA scores across all studies (reported immediately following Study 6).

Nevertheless, we found support for our hypothesis that SPA scores would mediate the association between trait pathogen avoidance and social bias. Indeed, SPA scores predicted biases against an obese target (replicating Study 1) and targets perceived as foreign, even when trait pathogen avoidance levels were not linked to these biases. For the bias against targets perceived as foreign, we found that White and Black participants who demonstrated higher SPA scores (compared to those who demonstrated lower scores) were more likely to perceive contagion in Asian or Latino faces compared to White or Black faces. The latter finding may reflect increased desire to avoid immigrants (i.e., increased ethnocentrism). Furthermore, SPA scores statistically mediated the association between germ aversion and both social biases. Taken together, these findings demonstrate the added value of measuring situational pathogen avoidance in addition to trait pathogen avoidance.

\section{Study 6}

In Study 6, we again examined whether social biases associated with pathogen avoidance are accounted for by SPA scores. We predicted that SPA scores would statistically mediate the effects of both trait pathogen avoidance and situationally activated pathogen avoidance on aversive reactions toward obese targets. We wanted to examine the effect of experimental manipulations of pathogen avoidance again because although we found support for such an effect in Studies $2-4$, it did not emerge in Study 5.

\section{Method}

\section{Participants}

Participants were undergraduate students $(N=198)$ recruited through the participant pool of a large, public university in the Southeastern US. We excluded 22 participants because they spent fewer than $10 \mathrm{~s}$ on the page with the manipulation article. One participant was 17, and their data were excluded. Our final sample included 175 participants $\left(M_{\text {age }}=18.64\right.$, $S D_{\text {age }}=1.37 ; 69 \%$ women; $95 \%$ heterosexual; $71 \%$ White/ Caucasian; $73 \%$ not Hispanic). Participants were fairly moderate in their political orientation $(M=5.47, S D=2.36)$ on a 1 (Very Conservative) to 10 (Very Liberal) scale and moderately religions $(M=48.57, \mathrm{SD}=31.69)$ on a 0 (Not religious at all) to 100 (Very religious) scale.

\section{Procedure}

Participants were randomly assigned to read a priming article about either the threat of an antibiotic-resistant infection or the threat of summer weather (as in Study 2). Immediately following the manipulation, participants completed the SPA scale $(\alpha=.83)$. Next, participants rated a photo of an obese person and reported on their gut-level evaluations (as in Studies 1 and 5). Finally, participants completed the PVD and provided demographic information.

\section{Results}

First, we examined whether experimental manipulation of pathogen threat increased SPA scores. As in Studies 2-4, participants who read an article about pathogen threat $(M=4.46$, $S D=1.15$ ) demonstrated higher SPA scores compared to participants who read an article about weather threat $(M=4.00$, 
$S D=0.99), t(173)=-2.82, p=.005, d=.43$. GA was not affected by the experimental manipulation, $t(174)=-1.31$; PI was marginally lower for participants in the pathogen threat condition $(M=3.68, S D=1.47)$ compared to the weather condition $(M=4.06, S D=1.41), t(174)=1.76, p=.080$. Next, we examined the zero-order correlations between the key variables: SPA, GA, PI, condition, and bias against the obese target. Bias against the obese target was significantly associated with SPA $(r=.27, p<.001)$, GA $(r=.22, p=.004)$, and PI $(r=.14, p=.072)$, but not condition $(r=-.04, p=.562)$. In multiple regression analyses, however, when bias against the obese target was regressed onto SPA, GA, PI, and condition, only the association with SPA scores was significant, $b=0.31$, $S E=0.12, t(170)=2.66, p=.008,95 \%$ CI $[0.08,0.55], s r-$ $=.19$ (all other $p$ 's $>.207)$.

Next, we turned to our main analyses. We first regressed SPA scores onto both GA and the experimental manipulation of pathogen threat. Both GA, $b=0.64, S E=.06, t(172)=$ $11.77, p<.001,95 \%$ CI [0.54, 0.75], $s r=.65$, and the experimental manipulation, $b=0.32, S E=.12, t(172)=2.63$, $p=.009,95 \%$ CI $[0.05,0.56], s r=.15$, were independently associated with higher SPA scores. Second, we estimated a mediational model using structural equation modeling in MPlus. We utilized this approach (as opposed to using PROCESS like in Study 5) in order to simultaneously estimate the indirect effects of GA and experimental manipulation through SPA scores. We report standardized effects (see Fig. 2). SPA scores were positively associated with aversive reactions toward the obese target. The direct effects between measures of pathogen avoidance and aversive reactions toward the obese target were not significant (GA: $b=0.05$, $S E=0.10, p=.581$; condition: $b=-0.11, S E=0.07$, $p=.147)$. However, as predicted, there was a significant indirect effect of GA through SPA scores, $b=0.20, S E=0.08$, $p=.017$, and simultaneously a marginally significant indirect effect of condition through SPA scores, $b=0.10, S E=0.06$, $p=.088$.

\section{Discussion}

Study 6 demonstrated that SPA scores were linked to both trait levels of pathogen avoidance and situationally activated pathogen avoidance. Indeed, over and above associations with trait pathogen avoidance, SPA scores were positively associated with bias against an obese target. Moreover, SPA scores mediated the associations between pathogen avoidance (both trait and situationally activated) and bias against an obese target. Thus, these findings and those from Study 5 highlight the benefit of using the SPA scale alongside measures of trait pathogen avoidance.

\section{Meta-analysis of the Experimental Manipulation Effects}

Because one goal of these studies was to develop a scale that responded to experimental manipulations of pathogen avoidance, we conducted a meta-analysis across the studies to provide a summary of the effect of priming pathogen threat on SPA scale scores. Following recommended procedures (Shadish and Haddock 1994), we first transformed the effect sizes from Studies 2-6 using the Fisher $r$-to- $z$ transformation (Borenstein 2009). Notably, for Study 2, we included the semi-partial $r$ effect size from the model which collapsed across, but controlled for, the two different priming methods $(r=.19, n=170)$. For Study 3, we included the semi-partial $r$ effect size from the association between condition and SPA scores $(r=.45, n=157)$. For Study 4, we included the effect size for the difference between the object and pathogen threat conditions, excluding the hand wipe condition $(r=.22, n=110)$. In order to be consistent, for Study 5, we included the effect size for the subsample of participants who completed the SPA scale directly after the manipulation $(r=.113, n=151)$. Finally, for Study 6 , we included the effect size for the experimental manipulation without controlling for GA $(r=.210, n=175)$. Because experimental studies in the pathogen avoidance literature use many different types of manipulations, we used a random-effects model using the Q-based estimate $(Q=$ 11.95 ) to obtain a summary effect size (transformed back into an $r$-based effect). Across the five samples, the meta-analytic findings suggest a robust, mediumsized positive effect of experimental manipulation on SPA scores, $r=.24,95 \%$ CI $[0.07,0.42]$. This is equal to a Cohen's $d$ of 49 .
Fig. 2 Path analysis model examining the statistical mediation of aversive reactions toward obese targets and both measures of pathogen avoidance (germ aversion and experimental condition) through the SPA scale

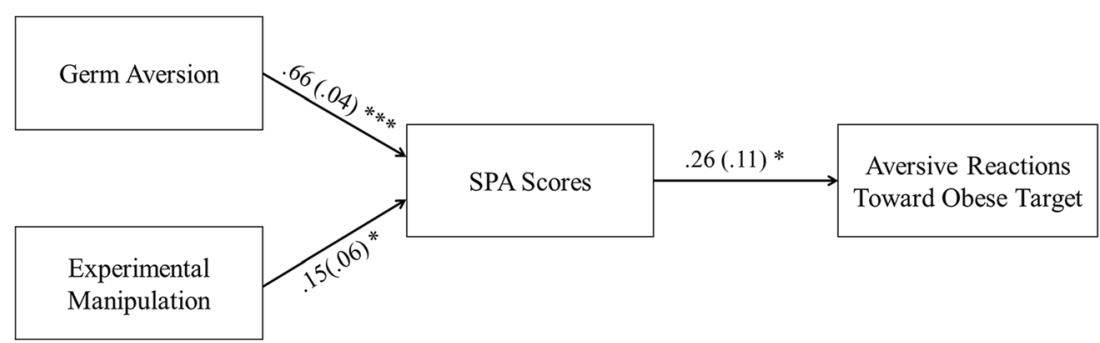




\section{Confirmatory Factor Analysis}

To confirm the factor structure of the SPA scale, we combined the data from Study 1 and the control conditions from the experimental studies 2-6 and performed a confirmatory factor analysis. ${ }^{12}$ A model with one latent factor had acceptable fit, $\chi^{2}(35)=229.87, p<.001$, RMSEA $=0.098$, SRMR $=0.056$, $\mathrm{CFI}=0.87$, and TLI $=0.84$. Factor loadings and descriptive statistics are presented in Table 1 . The two reverse-scored items have lower factor loadings, as is often the case with reverse-scored items (Barnette 2000; Weems and Onwuegbuzie 2001). Consistently, a model with a twofactor structure (with the two reverse-scored items loading on to second latent factor) had better model fit, $\chi^{2}(34)=$ $147.17, p<.001$, RMSEA $=0.076$, SRMR $=0.042, \mathrm{CFI}=$ 0.93 , and TLI $=0.90$. However, because from a theoretical standpoint both factors measure the same construct, we retained all 10 items in the scale.

\section{General Discussion}

Research on pathogen avoidance has increased steadily in the past decade and has linked pathogen avoidance to myriad psychological processes from attraction to xenophobia (Ackerman et al. 2018). Although reliable measurement scales exist for directly assessing trait pathogen avoidance, to date, the field has lacked a scale designed to directly assess situational fluctuations in pathogen avoidance, including those that follow experimental manipulations. In this paper, we developed and validated a scale - the Situational Pathogen Avoidance (SPA) scale - to address the need for such a measure.

Across five experiments (Studies 2-6), we examined whether various experimental manipulations of pathogen threat increased people's SPA scores. We also conducted a meta-analysis across the studies to provide a summary of the effect of pathogen threat on SPA scale scores. This metaanalysis documented a reliable effect of pathogen priming on SPA scores. Moreover, we demonstrated convergent, discriminant, and predictive validity of the SPA scale. SPA scores were associated with social biases against targets heuristically linked with pathogen threat. Indeed, associations between such biases and pathogen avoidance motives (both trait levels and situationally activated) were statistically mediated by the SPA scale. Overall, the SPA scale was associated with both trait and situationally activated pathogen avoidance

\footnotetext{
12 We additionally performed a CFA in a larger dataset that included the SPA scale (Makhanova and Shepherd 2020; Study 1) and found the fit indices somewhat improved: SRMR $=.041$, RMSEA $=.086$, TLI $=.899$, CFI $=.921$. Furthermore, a CFA of the PVD scale from the same study resulted in nearly identical fit indices: SRMR $=.045$, RMSEA $=.080$, $\mathrm{TLI}=.895, \mathrm{CFI}=.911$.
}

motives and with some of the key variables of interest in the pathogen avoidance literature.

Our results are in line with theoretical perspectives suggesting that environmental inputs affect the salience of chronic goals and individual differences, which consequently influence perceptions and behavior (e.g., McConnell 2011; Neuberg et al. 2011). In Study 6, we found that SPA scores reflect both chronic pathogen avoidance levels and acute environmental cues of increased pathogen threat. Furthermore, consistent with the hypothesis that pathogen avoidance processes influence perceptions and behavior through psychological states, trait pathogen avoidance, and environmental cues affected biases against an obese target through SPA scores. That is, biases against social targets are affected by both people's chronic pathogen avoidance levels as well as current information about pathogen threat, which are both captured by SPA scores. Thus, the SPA scale is a measurement tool that can complement the use of existing pathogen avoidance methodology.

The SPA scale contains specific features that make it uniquely valuable as a research tool. First, the scale emphasizes current, situational feelings. Both the overarching instructions and each item in the scale bring people's focus to how they are feeling right now. Second, the scale includes items that focus on affective (e.g., "Right now, if I was standing next to a person who sneezed I would feel disgusted") and behavioral (e.g., "Right now, I would try to sit on the opposite side of the room if I walked into the room where there was a person blowing their nose") responses to pathogens. Such items additionally emphasize the important context of social interactions. The SPA scale thus includes items that directly measure the desire for social distancing as a result of pathogen threat (e.g., "Right now, if someone coughed next to me without covering their mouth, I would move away from them). Because of these features, the SPA scale could be a valuable tool for researchers interested in processes at the intersection of pathogen avoidance and social behavior.

One way that researchers can use the SPA scale is to assess whether situational pathogen avoidance motives are associated with social processes. In this work, SPA scores were associated with two social biases: ethnocentrism and aversive reactions toward an obese target. One limitation of the obesity bias measure, however, was that it lacked a control target (i.e., someone of average weight) such as the non-immigrant group on the ethnocentrism measure in Study 5. Thus, rather than bias against a specific social target displaying a heuristic cue to illness, this association may reflect a general aversive reaction to social targets consistent with the link between pathogen avoidance and decreases in affiliative motivation (Sacco et al. 2014). Future research could directly test this distinction and whether SPA scores are negatively associated with affiliation. Furthermore, the SPA scale could also be associated with social processes beyond prejudice that reflect situationally 
activated pathogen avoidance, such as risk-aversion (Prokosch et al. 2019), moral vigilance (Murray et al. 2017), and heightened desire for improving one's appearance (Ackerman et al. 2018).

Another way that researchers can use the SPA scale is to examine whether and how situations influence pathogen avoidance motives. Although the present research validated the scale's function by assessing how experimental manipulations of pathogen threat increase SPA scores, pathogen avoidance is also affected by other situations such as being rejected (Sacco et al. 2014) or imagining oneself in a crowded place (Brown and Sacco 2020). Thus, researchers could use the SPA scale to assess how various situational factors and ecological variables (e.g., Sng et al. 2018) affect peoples' pathogen avoidance processes.

Yet another application for the SPA scale may be for research that tracks seasonal or longitudinal fluctuations in pathogen avoidance. For example, pathogen avoidance motives may increase during the flu season compared to other times of the year. Indeed, the authors have more recently collected data consistent with this hypothesis (Makhanova and Shepherd 2020). Data collected at the start of the COVID-19 pandemic demonstrated significantly higher SPA scores $(M=4.51, S D=$ 1.17) compared to the mean across the control conditions of samples reported in this manuscript $(M=4.03)$, one-sample $t(1586)=16.51, p<.001$. Scores were not higher for GA or PI at the start of the pandemic as compared to the scores collected for this manuscript. Furthermore, SPA scores significantly increased as the threat of the pandemic grew whereas neither GA nor PI scores increased. Another important longitudinal fluctuation in pathogen avoidance may occur around pregnancy and the post-partum period. Consistent with past research (Navarrete et al. 2007), SPA scores may increase when women become pregnant because mothers' immune systems become more vulnerable to pathogens and illness can have negative effects on fetal development. However, research has yet to examine how these processes develop over time within women and what happens in the post-partum period. SPA scores may remain elevated because mothers may be motivated to protect their newborns who do not have functional immune systems of their own. Alternatively, SPA scores may decrease because dealing with the newborns' bodily functions may desensitize mothers to potential sources of pathogens.

\section{Limitations and Other Future Directions}

One limitation of the SPA scale is that it does not directly address two other domains linked with the emotion of disgust-sexual and moral disgust (Tybur et al. 2009). Sexual and moral disgust predict important social behavior. Sexual and moral disgust, for example, are uniquely associated with agreeableness, conscientiousness, and lower psychopathy (Tybur et al. 2009). We designed the SPA scale to specifically focus on pathogen avoidance. Thus, although the
SPA scale represents a useful tool to examine how state fluctuations in pathogen avoidance are associated with social perceptions, it is not intended to measure state fluctuations in avoidance based on sexual or moral disgust.

The SPA scale is also not meant to replace existing measures of chronic pathogen avoidance-the Three Domain Disgust scale and the Perceived Vulnerability to Disease questionnaire - but rather to be a complementary tool that specifically assesses moment-to-moment changes in pathogen avoidance. Because the SPA scale focuses on social avoidance and disgust at the moment of administration, it cannot be relied upon as an individual difference measure of pathogen avoidance and, consistently, is correlated only moderately with individual measures of pathogen avoidance. Future research could examine whether individuals who perceive themselves to be more vulnerable to pathogen threat (i.e., report higher levels on the perceived infectability subscale of the PVD) may have stronger reactions to situations that connote increased contagion risk.

Overall, the use of complementary state and trait measurement tools may be especially useful for addressing several outstanding questions in the field of pathogen avoidance (see Tybur et al. 2014). One important area for future research is to examine when chronic versus situational changes in pathogen avoidance lead to similar social processes and when they lead to different social processes. For example, as mentioned previously, research has linked both chronic and situationally activated pathogen avoidance motives to social biases against targets heuristically linked with illness (e.g., Faulkner et al. 2004; Miller and Maner 2012). Chronic versus situational motives, however, may also lead to differing social processes and responses. Chronic pathogen avoidance, for example, has been linked with holding moral values that more strongly emphasize protecting one's ingroup from potential harm (van Leeuwen et al. 2017; Park and Isherwood 2011). Situationally activated pathogen avoidance, however, may not be associated with changes in one's moral values (Makhanova et al. 2019), but might instead facilitate stronger blame toward others who behave in ways that violate such group-protective values (Murray et al. 2017). Future research can benefit from using the SPA scale alongside existing pathogen avoidance scales to further examine parallel and divergent effects associated with chronic versus situational sources of pathogen avoidance. Furthermore, because the SPA scale focuses on threats from human interactions, it may be used in future research to examine whether situational pathogen avoidance is responsive to cues of nonzoonotic (pathogens that can be transmitted human-to-human) versus zoonotic (pathogens that can infect humans but are not transmitted human-to-human) pathogen presence (see Thornhill et al. 2010). 


\section{Conclusion}

Research on pathogen avoidance has grown exponentially in recent years. An impressive array of findings suggest that pathogen avoidance plays a central role in a number of important social psychological processes. The current research introduces a scale that captures situational fluctuations in pathogen avoidance and complements existing scales designed to measure chronic, trait-level pathogen avoidance. The current studies demonstrate that the SPA displays good reliability and validity, is responsive to experimental pathogen threat primes, and predicts a number of social biases known to result from pathogen avoidance. The SPA represents an important tool for researchers working in this literature.

\section{Compliance with Ethical Standards}

Conflict of Interest The authors declare that there is no conflict of interest.

\section{References}

Aarøe, L., Osmundsen, M., \& Petersen, M. B. (2016). Distrust as a disease avoidance strategy: individual differences in disgust sensitivity regulate generalized social trust. Frontiers in Psychology, 7, 1038.

Ackerman, J. M., Becker, D. V., Mortensen, C. R., Sasaki, T., Neuberg, S. L., \& Kenrick, D. T. (2009). A pox on the mind: disjunction of attention and memory in the processing of physical disfigurement. Journal of Experimental Social Psychology, 45(3), 478-485.

Ackerman, J. M., Tybur, J. M., \& Mortensen, C. R. (2018). Infectious disease and imperfections of self-image. Psychological Science, 29(2), 228-241.

Ainsworth, S. E., \& Maner, J. K. (2014). Hunger moderates the activation of psychological disease avoidance mechanisms. Evolutionary Behavioral Sciences, 8(4), 303-313.

Barnette, J. J. (2000). Effects of stem and Likert response option reversals on survey internal consistency: if you feel the need, there is a better alternative to using those negatively worded stems. Educational and Psychological Measurement, 60(3), 361-370.

Borenstein, M. (2009). Effect sizes for continuous data. In H. Cooper, L. V. Hedges, \& J. C. Valentine (Eds.), The handbook of research synthesis and meta-analysis (2nd ed.). New York: Russell Sage Foundation.

Brown, M., \& Sacco, D. F. (2016). Avoiding extraverts: pathogen concern downregulates preferences for extraverted faces. Evolutionary Psychological Science, 2, 278-286.

Brown, M., \& Sacco, D. F. (2020). How and when crowd salience activates pathogen-avoidant motives. Evolutionary Behavioral Sciences. Advance online publication. https://doi.org/10.1037/ebs0000191.

Brown, M., Rodriguez, D. N., Gretak, A. P., \& Berry, M. A. (2017). Preliminary evidence for how the behavioral immune system pre-dicts juror decision-making. Evolutionary Psychological Science, 3, 325334.

Brown, M., Keefer, L. A., Sacco, D. F., \& Bermond, A. (2019). Is the cure a wall? Behavioral immune system responses to a disease metaphor for immigration. Evolutionary Psychological Science, 5(3), 343-356.

Burns, G. L., Keortge, S. G., Formea, G. M., \& Sternberger, L. G. (1996). Revision of the Padua inventory of obsessive compulsive disorder symptoms: distinctions between worry, obsessions, and compulsions. Behaviour Research and Therapy, 34(2), 163-173.
Cacioppo, J. T., Petty, R. E., \& Feng Kao, C. (1984). The efficient assessment of need for cognition. Journal of Personality Assessment, 48(3), 306-307.

Crandall, C. S. (1994). Prejudice against fat people: ideology and self-interest. Journal of Personality and Social Psychology, 66(5), 882-894.

Duncan, L. A., \& Schaller, M. (2009). Prejudicial attitudes toward older adults may be exaggerated when people feel vulnerable to infectious disease: evidence and implications. Analyses of Social Issues and Public Policy, 9(1), 97-115.

Duncan, L. A., Schaller, M., \& Park, J. H. (2009). Perceived vulnerability to disease: development and validation of a 15 -item self-report instrument. Personality and Individual Differences, 47(6), 541-546.

Faulkner, J., Schaller, M., Park, J. H., \& Duncan, L. A. (2004). Evolved disease-avoidance mechanisms and contemporary xenophobic attitudes. Group Processes \& Intergroup Relations, 7(4), 333-353.

Fincher, C. L., \& Thornhill, R. (2012). Parasite-stress promotes in-group assortative sociality: the cases of strong family ties and heightened religiosity. Behavioral and Brain Sciences, 35(2), 61-79.

Gosling, S. D., Rentfrow, P. J., \& Swann Jr., W. B. (2003). A very brief measure of the Big-Five personality domains. Journal of Research in Personality, 37(6), 504-528.

Haidt, J., McCauley, C., \& Rozin, P. (1994). Individual differences in sensitivity to disgust: a scale sampling seven domains of disgust elicitors. Personality and Individual Differences, 16(5), 701-713.

Haselton, M. G., \& Nettle, D. (2006). The paranoid optimist: an integrative evolutionary model of cognitive biases. Personality and Social Psychology Review, 10(1), 47-66.

Hu, L. T., \& Bentler, P. (1998). Fit indices in covariance structure modeling: sensitivity to underparameterized model misspecification. Psychological Methods, 3(4), 424-453.

Huang, J. Y., Sedlovskaya, A., Ackerman, J. M., \& Bargh, J. A. (2011). Immunizing against prejudice: effects of disease protection on attitudes toward out-groups. Psychological Science, 22(12), 1550-1556.

Karinen, A. K., Molho, C., Kupfer, T. R., \& Tybur, J. M. (2019). Disgust sensitivity and opposition to immigration: does contact avoidance or resistance to foreign norms explain the relationship? Journal of Experimental Social Psychology, 84, 103817.

Lakens, D., \& Etz, A. J. (2017). Too true to be bad: When sets of studies with significant and nonsignificant findings are probably true. Social Psychological and Personality Science, 8(8), 875-881.

Lieberman, D. L., Tybur, J. M., \& Latner, J. D. (2012). Disgust sensitivity, obesity stigma, and gender: contamination psychology predicts weight bias for women, not men. Obesity, 20(9), 1803-1814.

Lund, E. M., \& Miller, S. L. (2014). Is obesity un-American? Disease concerns bias implicit perceptions of national identity. Evolution and Human Behavior, 35(4), 336-340.

MacCallum, R. C., Browne, M. W., \& Sugawara, H. M. (1996). Power analysis and determination of sample size for covariance structure modeling. Psychological Methods, 1(2), 130-149.

Makhanova, A., \& Shepherd, M. A. (2020). Behavioral immune system linked to responses to the threat of COVID-19. Personality and Individual Differences, 110221.

Makhanova, A., Miller, S. L., \& Maner, J. K. (2015). Germs and the outgroup: chronic and situational disease concerns affect intergroup categorization. Evolutionary Behavioral Sciences, 9(1), 8-19.

Makhanova, A., Plant, E., Monroe, A., \& Maner, J. (2019). Binding together to avoid illness: pathogen avoidance and moral worldviews. Evolutionary Behavioral Sciences, 13(2), 182-204.

McConnell, A. R. (2011). The multiple self-aspects framework: selfconcept representation and its implications. Personality and Social Psychology Review, 15(1), 3-27.

McNeish, D., An, J., \& Hancock, G. R. (2018). The thorny relation between measurement quality and fit index cutoffs in latent variable models. Journal of Personality Assessment, 100(1), 43-52. 
Miller, S. L., \& Maner, J. K. (2012). Overperceiving disease cues: the basic cognition of the behavioral immune system. Journal of Personality and Social Psychology, 102(6), 1198-1213.

Mortensen, C. R., Becker, D. V., Ackerman, J. M., Neuberg, S. L., \& Kenrick, D. T. (2010). Infection breeds reticence: the effects of disease salience on self-perceptions of personality and behavioral avoidance tendencies. Psychological Science, 21(3), 440-447.

Murray, D. R., \& Schaller, M. (2012). Threat (s) and conformity deconstructed: perceived threat of infectious disease and its implications for conformist attitudes and behavior. European Journal of Social Psychology, 42(2), 180-188.

Murray, D. R., Kerry, N., \& Gervais, W. M. (2017). On disease and deontology: multiple tests of the influence of disease threat on moral vigilance. Social Psychological and Personality Science, 1948550617733518.

Navarrete, C. D., \& Fessler, D. M. T. (2006). Disease avoidance and ethnocentrism: the effects of disease vulnerability and disgust sensitivity on intergroup attitudes. Evolution and Human Behavior, 27(4), 270-282.

Navarrete, C. D., Fessler, D. M., \& Eng, S. J. (2007). Elevated ethnocentrism in the first trimester of pregnancy. Evolution and Human Behavior, 28(1), 60-65.

Neuberg, S. L., Kenrick, D. T., \& Schaller, M. (2011). Human threat management systems: self-protection and disease avoidance. Neuroscience \& Biobehavioral Reviews, 35(4), 1042-1051.

Neuliep, J. W., \& McCroskey, J. C. (1997). The development of a US and generalized ethnocentrism scale. Communication Research Reports, 14(4), 385-398.

Oaten, M. J., Stevenson, R. J., \& Case, T. I. (2017). Compensatory upregulation of behavioral disease avoidance in immunocompromised people with rheumatoid arthritis. Evolution and Human Behavior, 38(3), 350-356.

Olatunji, B. O., Williams, N. L., Tolin, D. F., Abramowitz, J. S., Sawchuk, C. N., Lohr, J. M., \& Elwood, L. S. (2007). The disgust scale: item analysis, factor structure, and suggestions for refinement. Psychological Assessment, 19(3), 281-297.

Park, J. H., \& Isherwood, E. (2011). Effects of concerns about pathogens on conservatism and anti-fat prejudice: are they mediated by moral intuitions? The Journal of Social Psychology, 151(4), 391-394.

Park, J. H., Schaller, M., \& Crandall, C. S. (2007). Pathogen-avoidance mechanisms and the stigmatization of obese people. Evolution and Human Behavior, 28(6), 410-414.

Paulhus, D. L. (1998). Balanced inventory of desirable responding (BIDR). Acceptance and Commitment Therapy. Measures Package, 41.

Prokosch, M. L., Gassen, J., Ackerman, J. M., \& Hill, S. E. (2019). Caution in the time of cholera: pathogen threats decrease risk tolerance. Evolutionary Behavioral Sciences, 13(4), 311-334.

Rozin, P., \& Fallen, A. E. (1987). A perspective on disgust. Psychological Review, 94(1), 23-41.

Sacco, D. F., Young, S. G., \& Hugenberg, K. (2014). Balancing competing motives: adaptive trade-offs are necessary to satisfy disease avoidance and interpersonal affiliation goals. Personality and Social Psychology Bulletin, 40(12), 1611-1623.

Schaller, M. (2014). When and how disgust is and is not implicated in the behavioral immune system. Evolutionary Behavioral Sciences, 8(4), 251-256.

Schaller, M., \& Park, J. H. (2011). The behavioral immune system (and why it matters). Current directions in psychological science, 20(2), 99-103.

Schaller, M., Miller, G. E., Gervais, W. M., Yager, S., \& Chen, E. (2010). Mere visual perception of other people's disease symptoms facilitates a more aggressive immune response. Psychological Science, 21(5), 649-652.
Shadish, W. R., \& Haddock, C. K. (1994). Combining estimates of effect size. In H. Cooper \& L. V. Hedges (Eds.), The handbook of research synthesis (pp. 261-281). New York: Russell Sage Foundation.

Shook, N. J., Terrizzi Jr., J. A., Clay, R., \& Oosterhoff, B. (2015). In defense of pathogen disgust and disease avoidance: a response to Tybur et al.(2015). Evolution and Human Behavior, 36(6), 498 502.

Sng, O., Neuberg, S., Varnum, M. E., \& Kenrick, D. (2018). The behavioral ecology of cultural psychological variation. Psychological Review, 125(5), 714-743.

Stanley, L. M., \& Edwards, M. C. (2016). Reliability and model fit. Educational and Psychological Measurement, 76(6), 976-985.

Terrizzi Jr., J. A., Shook, N. J., \& Ventis, W. L. (2010). Disgust: a predictor of social conservatism and prejudicial attitudes toward homosexuals. Personality and Individual Differences, 49(6), 587-592.

Terrizzi Jr., J. A., Shook, N. J., \& McDaniel, M. A. (2013). The behavioral immune system and social conservatism: a meta-analysis. Evolution and Human Behavior, 34(2), 99-108.

Thornhill, R., Fincher, C. L., Murray, D. R., \& Schaller, M. (2010). Zoonotic and non-zoonotic diseases in relation to human personality and societal values: support for the parasite-stress model. Evolutionary Psychology, 8(2), 147470491000800201.

Tybur, J. M., \& de Vries, R. E. (2013). Disgust sensitivity and the HEXACO model of personality. Personality and Individual Differences, 55(6), 660-665.

Tybur, J. M., Lieberman, D., \& Griskevicius, V. (2009). Microbes, mating, and morality: individual differences in three functional domains of disgust. Journal of Personality and Social Psychology, 97(1), 103-122.

Tybur, J. M., Frankenhuis, W. E., \& Pollet, T. V. (2014). Behavioral immune system methods: surveying the present to shape the future. Evolutionary Behavioral Sciences, 2014(8), 274-283.

Tybur, J. M., Inbar, Y., Güler, E., \& Molho, C. (2015a). Is the relationship between pathogen avoidance and ideological conservatism explained by sexual strategies? Evolution and Human Behavior, 36(6), 489-497.

Tybur, J. M., Inbar, Y., Güler, E., \& Molho, C. (2015b). Pathogen disgust requires no defense: a response to Shook, Terrizzi, Clay, \& Oosterhoff (2015). Evolution and Human Behavior, 36(6), 502-504.

Tybur, J. M., Inbar, Y., Aarøe, L., Barclay, P., Barlow, F. K., De Barra, M., et al. (2016). Parasite stress and pathogen avoidance relate to distinct dimensions of political ideology across 30 nations. Proceedings of the National Academy of Sciences, 113(44), 12408-12413.

van Leeuwen, F., \& Petersen, M. B. (2018). The behavioral immune system is designed to avoid infected individuals, not outgroups. Evolution and Human Behavior, 39(2), 226-234.

van Leeuwen, F., Dukes, A., Tybur, J., \& Park, J. (2017). Disgust sensitivity relates to moral foundations independent of political ideology. Evolutionary Behavioral Sciences, 11(1), 92-98.

Wang, I. M., \& Ackerman, J. M. (2019). The infectiousness of crowds: crowding experiences are amplified by pathogen threats. Personality and Social Psychology Bulletin, 45(1), 120-132.

Weems, G. H., \& Onwuegbuzie, A. J. (2001). The impact of midpoint responses and reverse coding on survey data. Measurement and Evaluation in Counseling and Development, 34(3), 166-176.

Young, S. G., Sacco, D. F., \& Hugenberg, K. (2011). Vulnerability to disease is associated with a domain-specific preference for symmetrical faces relative to symmetrical non-face stimuli. European Journal of Social Psychology, 41(5), 558-563.

Publisher's Note Springer Nature remains neutral with regard to jurisdictional claims in published maps and institutional affiliations. 